\title{
Erratum to: Effect of solid freeform fabrication-based polycaprolactone/poly(lactic-co-glycolic acid)/collagen scaffolds on cellular activities of human adipose-derived stem cells and rat primary hepatocytes
}

\author{
Jin-Hyung Shim • Arthur Joon Kim • \\ Ju Young Park $\cdot$ Namwoo Yi $\cdot$ Inhye Kang \\ Jaesung Park $\cdot$ Jong-Won Rhie $\cdot$ Dong-Woo Cho
}

Published online: 4 May 2013

(C) Springer Science+Business Media New York 2013

Erratum to: J Mater Sci: Mater Med

(2013) 24:1053-1065.

DOI 10.1007/s10856-013-4867-8

The authors omitted to note that Jin-Hyung Shim and Arthur Joon Kim should be recognized as co-first authors for their equal contribution to this article.

Jin-Hyung Shim and Arthur Joon Kim are first co-authors.

The online version of the original article can be found under doi:10.1007/s10856-013-4867-8.

J.-H. Shim · N. Yi · I. Kang · J. Park - D.-W. Cho ( () Department of Mechanical Engineering, Pohang University of Science and Technology (POSTECH), San 31 Hyoja-dong Nam-gu, Pohang, Gyungbuk 790-784, South Korea e-mail: dwcho@postech.ac.kr

\section{A. J. Kim}

Harvard School of Dental Medicine, 188 Longwood Avenue,

Boston, MA 02115, USA

J. Y. Park · D.-W. Cho

Division of Integrative Biosciences and Biotechnology,

Pohang University of Science and Technology (POSTECH),

San 31 Hyoja-dong Nam-gu, Pohang, Gyungbuk 790-784,

South Korea

J.-W. Rhie

Department of Plastic Surgery, College of Medicine,

The Catholic University of Korea, Bucheon, South Korea 